\title{
EMPODERAMIENTO DE MUJERES EN PROGRAMAS Y PROYECTOS DE SEGURIDAD ALIMENTARIA EN SIETE COMUNIDADES DEL MUNICIPIO DE SAN JOSÉ DE CUSMAPA, MADRÍZ, 2006 - 2008
}

\section{WOMEN EMPOWERMENT IN PROGRAMS AND PROJECTS OF FOOD SECURITY IN SEVEN COMMUNITIES OF THE MUNICIPALITY OF SAN JOSE DE CUSMAPA, MADRIZ, 2006 - 2008}

\section{Rivera López Francisco Javier ${ }^{1}$, Báez Gómez Angélica María ${ }^{2}$}

${ }^{1}$ Licenciado en Desarrollo Rural, Universidad Nacional Agraria, Managua, Nicaragua. km 12 1/2 carretera norte, e-mail: franbrisa@yahoo.com ${ }^{2}$ MSc. Docente de la Facultad de Desarrollo Rural. FDR-UNA.

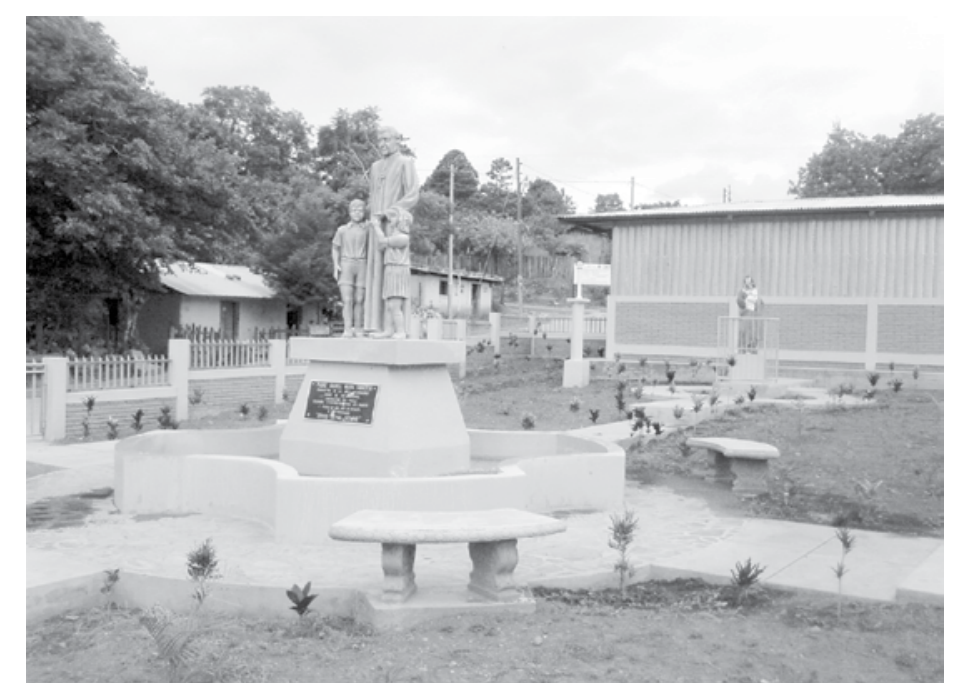

\section{RESUMEN}

El estudio se realizó en siete comunidades del municipio de San José de Cusmapa, ubicado en el Departamento de Madríz. Este municipio se ubica a $34 \mathrm{~km}$ al suroeste de la cabecera departamental Somoto, y a $274 \mathrm{~km}$ al norte de Managua. El objetivo del estudio fue analizar los factores sociales que influyen en el nivel de empoderamiento de las mujeres en los programas y proyectos de Desarrollo Rural en la Seguridad Alimentaria, implementados en siete comunidades del municipio de San José de Cusmapa. La metodología se realizó en tres fases. La primera fase consistió en la selección de la unidad de análisis y población en estudio, conformada por las comunidades San Francisco de Imire, Ángel 1, Ángel 2, El Mamey, Los Llanitos, El Mojón y El Apante. Estas comunidades fueron las que trabajaron con programas y proyectos de seguridad alimentaria y nutricional en el Municipio. Para la selección de la muestra se utilizó el muestreo de sujetos tipos. La revisión bibliográfica consistió en la recopilación de información y depuración de la misma
This study was conducted in seven communities at San Jose; Cusmapa municipality, located in the North part of the country, in Madriz department, 34 kilometers southwest from Somoto, the main city of the department, and $274 \mathrm{~km}$ North apart from Managua, Republic of Nicaragua. This research aims to analyze the main social factors that influence the level of empowerment of women in programs and projects Rural Development Food Security implemented in seven communities in San José Cusmapa municipality. The methodology was implemented in three phases: first phase consisted of selecting the unit of analysis and study population comprised of St. Joseph County communities of Cusmapa: San Francisco de Imire, Angel 1, Angel 2, El Mamey, The Llanitos, The Marker and The Apante, these communities were chosen because they are the ones who worked with programs and projects for food security and nutrition in the Borough, for selecting the sample of subjects using the sampling rate, the literature review consisted of collection and treatment 
en base a los intereses de la investigación. La segunda fase consistió en la recolección, procesamiento y análisis de la información. El levantamiento de los datos primarios se realizó por medio de talleres comunitarios, entrevistas, observación, árbol de problemas, informantes claves y caracterización municipal. Para el procesamiento de los datos primarios se diseñaron tablas de salida para agrupar la información ordenándolos según las variables planteadas. El análisis de la información permitió ubicar las comunidades en escenarios de niveles de empoderamiento. La tercera fase consistió en la elaboración del informe final, del cual se redacto un primer borrador, un segundo borrador que permitió validar y hacer las correspondientes correcciones para la elaboración del informe final. El análisis de los factores sociales que influyen en el nivel de empoderamiento de las mujeres y las características socioculturales de las comunidades permitió la ubicación de cada una de las comunidades en escenarios: I escenario óptimo para el empoderamiento, II escenario con oportunidad para el empoderamiento, III escenario con limitaciones para el empoderamiento y IV escenario con extremas limitaciones para el empoderamiento. La organización, capacitación, comunicación y participación en las comunidades son factores que limitan la toma de decisiones en los asuntos que ponen en riesgo el desarrollo de las familias. Se recomienda la interacción entre instituciones para fortalecer acciones en pro del desarrollo de las comunidades, el trabajo de desarrollo organizativo a nivel de agencia y comunidad, y la creación de redes que le permita a los comunitarios ser autogestionarios. La capacitación debe realizarse en función de la efectiva participación de los comunitarios, de manera que permita en mediano y largo plazo la implementación de técnicas en seguridad alimentaria. Palabras clave: Mujeres, empoderamiento, participación, comunicación. Organización. Capacitación. information based on the same research interests. The second phase consisted of the collection, processing and information analysis, the lifting of the primary data was conducted through community workshops, interviews, observation, problem tree, key informants and local characterization, to process raw data obtained output tables were designed to group information according to the variables proposed sorting, analysis of information to the placing of the communities in stages of levels of empowerment. The third phase consisted of preparing the final report, which compose a first draft, a second draft that allowed validation and make appropriate corrections for final reporting. The analysis of social factors that influence the level of empowerment of women allowed the location of each of the communities in stages: Stage I optimal Empowerment, II Stage with opportunity for Empowerment, with limited Stage III Empowerment and Stage IV with extreme limitations for empowerment, this according to the social-cultural characteristics of communities. The organization, training, communication and participation in the communities are factors that limit the decision-making in matters which threaten the development of families. It is recommended that interaction between institutions to strengthen actions for community development work the issue of organizational development agency and community level, creating networks that will enable the community to be self-managed. Training should be based on the effective participation of community in order to allow medium and long term technical implementation of food security.

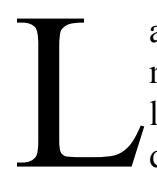

a inseguridad alimentaria es una de las problemáticas mas frecuentes en Nicaragua, permaneciendo en la lista de países de bajos ingresos y con déficit de alimentos, elaborada por la Organización de las Naciones Unidas para la Agricultura y la Alimentación, FAO, junto a otros ochenta y un país del mundo.

Para Nicaragua, estos datos corresponden a 1.5 millones de personas, formando así también parte de los 25 países en el mundo, que padecen de inseguridad alimentaria y nutricional. Y, en el caso del bajo peso ${ }^{1}$, corresponde al $10 \%$ de los niños y niñas menores de cinco años (FAO. 2004).

El municipio de San José de Cusmapa es el más pobre de Nicaragua en el se encuentran diferentes actores que desarrollan acciones para contribuir a la seguridad alimentaria y nutricional los cuales en su mayoría se encuentran en la parte interna del municipio y el resto con sede en Somoto, también se encuentran otras organizaciones que hacen

${ }^{1}$ Bajo peso se refiere a $33.5 \mathrm{lb}$ en niños menores de 5 años en comparación al peso normal (46.5 lb), según datos del PMA citado por PRESANCA presencia en lo externo de forma indirecta en el municipio cuyo trabajo es a través de la co-ejecución como es el caso de el Programa Mundial de Alimentos PMA (PRESANCA. 2007).

Según PMA. 2005, el apoyo a las mujeres y sus iniciativas desde una perspectiva emancipadora constituyen un elemento estratégico. El empoderamiento de las mujeres se debe en particular a los proyectos que incentiven a la Seguridad Alimentaria; producción; capacitaciones; apoyo a colectivos organizados de mujeres y créditos a la producción y a microempresas. En el caso de las acciones de emergencia, se fomenta la participación de las mujeres en la recepción y distribución de la ayuda alimenticia y de bienes de consumo, fortaleciendo su función en la organización comunitaria.

En las comunidades del municipio de San Jose de Cusmapa, se desconocen los factores socioculturales (participación, comunicación, organización y capacitación) que influyen en el nivel de empoderamiento de las mujeres 
en los programas y proyectos de Seguridad Alimentaria. En este contexto la Universidad Politécnica de Madrid, España y la Universidad Nacional Agraria, Nicaragua pretenden mediante la puesta en marcha del proyecto "Incidencia de los proyectos y programas de Desarrollo Rural en la Seguridad Alimentaria y Nutricional de las comunidades rurales de Nicaragua" fortalecer la investigación y las acciones de lucha contra el hambre en los procesos de Desarrollo Rural y la incidencia de las mujeres en proyectos y programas de Seguridad Alimentaria, razón por la que se realizó la presente investigación.

\section{MATERIALES Y MÉTODOS}

Diseño metodológico. Por ser de naturaleza cualitativa y debido a los objetivos definidos, la investi-gación fue de tipo descriptivo. (Piura, 1994). En el presente estudio dentro del proceso metodológico se realizaron diferentes fases, las cuales se detallan a continuación:

\section{FASE I. Selección de la unidad de análisis y población en estudio}

Paso 1. Selección de la unidad de análisis. La selección del área en estudio estuvo conformada por comunidades del Municipio San José de Cusmapa: San Francisco de Imire, Ángel 1, Ángel 2, El Mamey, Los Llanitos, El Mojón y El Apante. Estas comunidades fueron elegidas por que son las que trabajaron con programas y proyectos de seguridad alimentaria y nutricional en el Municipio. Dentro de la unidad de análisis los protagonistas de la información recopilada se encuentran como eje principal las mujeres comunitarias quienes fueron beneficiadas por los proyectos, técnicos que dieron seguimiento al proceso de los programas $\mathrm{y}$ proyectos durante el período 2006 al 2008 e instituciones que ejecutaron los programas y proyectos en las comunidades, siendo estos: Acción contra el hambre, MAGFOR-Hambre Cero, Movimiento Comunal Nicaragüense, MINSA, UNICAM-INSFOP, PROSUD, Fundación Padre Fabretto.

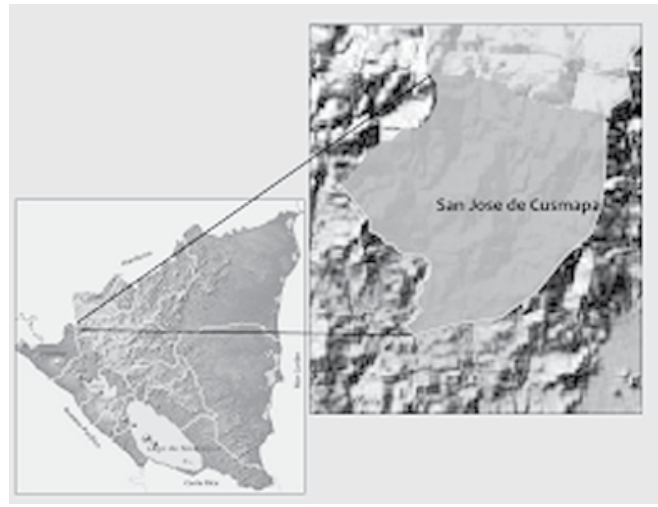

Figura 1. Mapa de área en Estudio

Esta unidad de análisis se tomó con el fin de conocer el nivel de toma de decisiones de las mujeres dentro de los programas y proyectos ejecutados en el municipio de San José de Cusmapa por organismos e instituciones que trabajan en pro de la seguridad alimentaria enfocada a igualdad de género.

Paso 2. Recolección de datos secundarios. Revisión bibliográfica. La recopilación bibliográfica más relevante fue la de libros con información del municipio, revistas, tesis relacionada al tema e información documental como son archivos electrónicos facilitados por las entidades en estudio.

Paso 3. Definición de la muestra. Para la selección de la muestra se utilizó el muestreo de sujetos tipos que es recomen dada para investigaciones cualitativas, particularmente las que se usan como instrumentos las entrevistas (Piura, 1994).

Se utilizaron como criterios para la selección de la muestra; la cantidad de Organismo e Instituciones con incidencia en el municipio en programas y proyectos SAN, técnicos y mujeres comunitarias con conocimientos sobre el tema, habilidades de liderazgo y tiempo disponible, comunidadesaccesibles, mujeres beneficiariasporlosproyectos y programas SAN, informantes claves (maestros, estudiantes, comunitarios, enfermeras, técnico de la alcaldía municipal) (Tabla 1).

Tabla 1. Muestra en estudio

\begin{tabular}{ll}
\hline Programa/Proyecto & Comunidades \\
\hline 1. Ministerio de Salud (MINSA). & Los Llanitos \\
2. Universidad Campesina/ Instituto de & El Mojón \\
Formación Permanente (UNICAM/INSFOP). & \\
3. Proyectos de Sudamérica (PROSUD). & El Apante \\
4 Fundación Padre Fabretto. & El Mamey \\
5. Acción Contra el Hambre/PRESANCA, & San Francisco de Imire \\
6. MAGFOR/hambre Cero & Ángel 1 \\
7. Movimiento Comunal Nicaragüense (MCN). & Ángel 2
\end{tabular}

En cada comunidad se involucró una mujer facilitadora y dos mujeres comunitarias

Informantes claves. Tres mujeres comunitarias, 1 profesor, 2 estudiantes, 1 técnico de base de la alcaldía municipal, Alcaldesa municipal, Doctora del MINSA y Directora de la Fundación Padre Fabretto, para un total de 10. 
FASE II. Recolección, procesamiento y análisis Paso 1. Recolección de datos primarios. El levantamiento de los datos primarios se realizó por medio de talleres comunitarios con la presencia de técnicos de las instituciones involucradas en los proyectos de seguridad alimentaria y nutricional, participación de mujeres comunitarias y mujeres evaluadoras de cada una de las comunidades atendidas por las instituciones presentes en el municipio (San Francisco de Imire, ángel 1, ángel 2, el Mamey, El apante, El mojón y los llanitos). Para estos talleres se utilizaron diversas técnicas como son: árbol de problemas, observación participante y entrevistas, además se recopilo documentación existente en el municipio entre ellos la caracterización que PRESANCA realizó en el año 2007 relacionado a los proyectos.
Entrevista individual. Para la realización de las entrevistas se retomaron los tópicos en los que se centro el estudio: tipos de participación, redes organizacionales, niveles de comunicación y niveles de capacitación, todos estos factores enmarcados en el nivel de empoderamiento de las mujeres en los programas y proyectos de seguridad alimentaria.

Árbol de problemas. Se utilizó el árbol de problemas en dos talleres para conocer el problema principal que cada comunidad presenta en función de la inseguridad alimentaria y nutricional, por tanto se consiguió realizar un taller con técnicos y mujeres evaluadoras y otro con mujeres comunitarias a evaluarse con el fin de verificar la información. Las mujeres facilitaron la entrevista participativa por productoras (EPP), las cuales se realizaron de forma que, las beneficiarias de un proyecto entrevistaran a beneficiarias de otros proyectos (Figura 2).

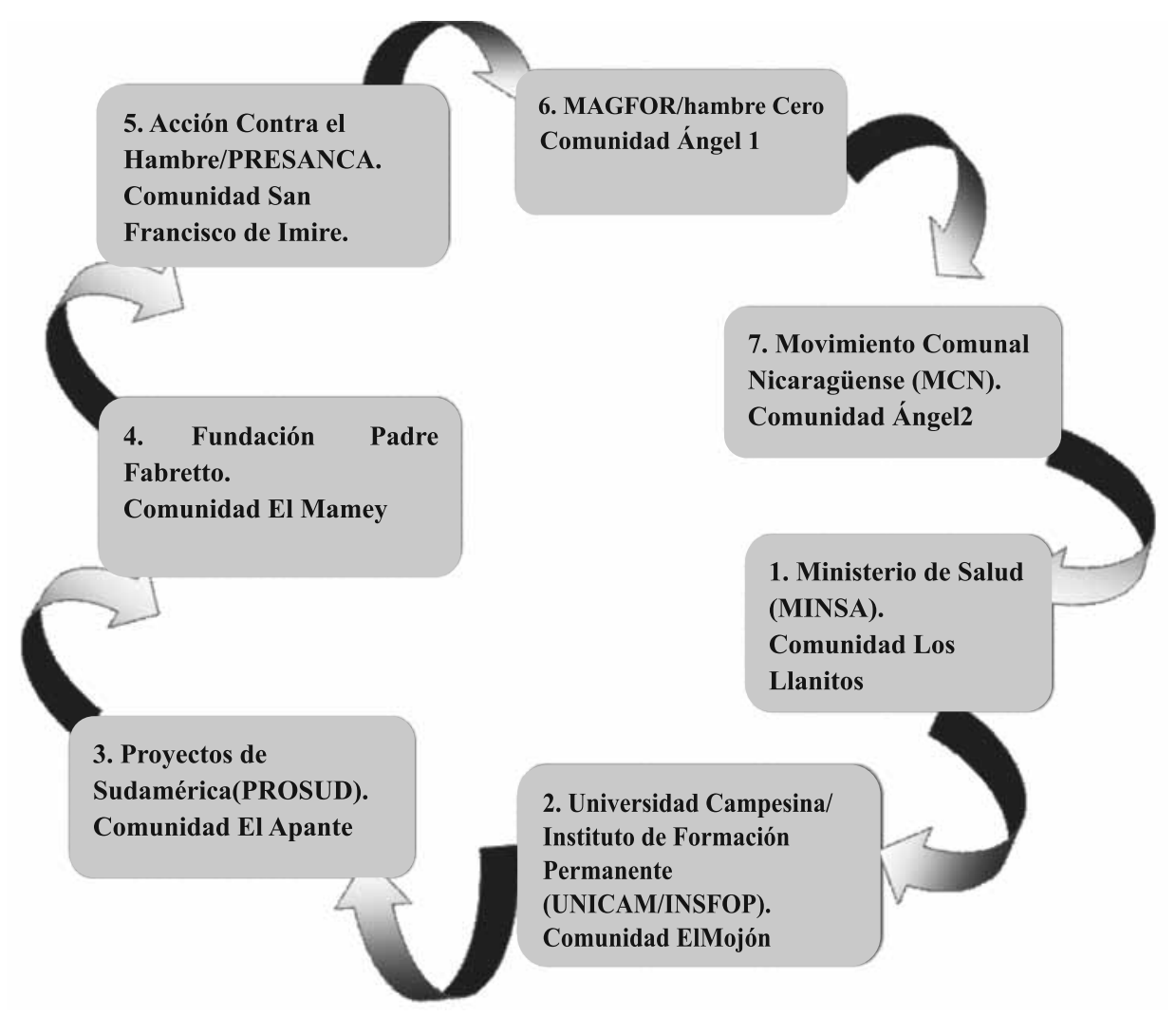

Figura 2. Entrevista Participativa por Productoras (EPP Externa) Las flechas indican el orden de las entrevistas, realizadas entre mujeres comunitarias beneficiadas por los programas y proyectos de seguridad alimentaria. Elaboración propia. 
Mapa comunitario. En el presente estudio se tomó como referencia el mapa base del municipio de San José de Cusmapa, elaborado por la alcaldía municipal, el cual sirvió como referencia para la selección de las comunidades en estudio tomando como uno de los criterios para la selección de la muestra la distancia de estas en relación al casco urbano, de igual manera para delimitar el area de estudio.

Observación participante. Las visitas y estadía durante un mes en las comunidades permitió realizar las observaciones necesarias para conocer, entender y discernir sobre la situación de seguridad alimentaria y nutricional en las familias, participando con ética, establecimiento de buenas relaciones y selección de informantes claves para determinar en conjunto qué y donde observar. La participación en el taller ejecutado por parte de UNICAM en la comunidad Los Llanitos y El Mojón sobre escuelas de campo, elaboración y transformación de productos de la comunidad El Mamey asistida por Fundación Padre Fabretto y capacitación sobre el bono productivo en la comunidad Ángel 1 realizado por MAGFOR/Hambre Cero. En todo el proceso se utilizó la libreta de campo para recepcionar la información suministrada por los informantes claves y percibidas por el investigador.

Taller. Para el presente estudio se realizaron dos talleres en el casco urbano del municipio de San José de Cusmapa. El primer taller, el cual tuvo una duración de un día, se realizó con el propósito de definir los criterios de selección para la aplicación de entrevistas participativas por productoras, esto en conjunto con los técnicos que trabajan en programas y proyectos de seguridad alimentaria y mujeres líderes. En este taller los técnicos territoriales se apropiaron del proceso a seguir y contribuyeron al ajuste metodológico de la guía, basándose en los criterios establecidos y la apreciación de las lideres, revisando las herramientas a utilizar en la recolección de la información en las comunidades, se mejoró el contenido de la entrevista y se adaptó al lenguaje a una forma sencilla para que las mujeres a entrevistar la entendieran con facilidad, la participación del equipo técnico de campo y mujeres líderes fue clave para brindar la información necesaria sobre las comunidades atendidas, cantidad, nombre y ubicación de las mujeres sobre los que se efectuó la selección.

Paso 2. Procesamiento y análisis de datos colectados en campo. Para el procesamiento de los datos primarios obtenidos de los talleres comunitarios y las entrevistas, se diseñaron tablas de salida para agrupar la información; luego se procesaron los datos obtenidos, ordenando la información

El análisis de la información permitió ubicar a las comunidades en escenarios de niveles de empoderamiento, tomando como referencia los productos esperados de las variables planteadas (Tabla 2), como son:

Triangulación de la información. Se asume convencionalmente que la triangulación es el uso de múltiples métodos en el estudio de un mismo objeto. Esta es la definición genérica, pero es solamente una forma de la estrategia. Es conveniente concebir la triangulación envolviendo variedades de datos, investigadores y teorías, así como metodologías.

Para Cowman (2001), la triangulación se define como la combinación de múltiples métodos en un estudio del mismo objeto o evento para abordar mejor el fenómeno que se investiga, como es el caso de la presente investigación.

Por su parte, Morse. 2002, define la triangulación metodológica como el uso de al menos dos métodos, usualmente cualitativo y cuantitativo para direccionar el mismo problema de investigación. Cuando un método singular de investigación es inadecuado, la triangulación se usa para asegurar que se toma una aproximación más comprensiva en la solución del problema de investigación.

Sin embargo se retoman los aspectos de Morse en el sentido del uso de métodos cualitativos para abordar el problema de investigación.

Tabla 2. Escenarios de niveles de empoderamiento

\begin{tabular}{lll}
$\begin{array}{l}\text { II Escenario con oportunidad para el } \\
\text { empoderamiento }\end{array}$ & I Escenario óptimo para el empoderamiento \\
\hline$>\quad$ Participación interactiva & $>\quad$ Participación por auto movilización \\
$>\quad$ Participación orientada hacia la acción & $>\quad$ Comunicación personal \\
$>\quad$ Comunicación impersonal - automática & $>\quad$ Ned de organización formal \\
$>\quad$ Nivel de capacitación intermedio & III Escenario con limitaciones para el \\
$>\quad$ Red de organización orgánica & empoderamiento \\
\hline IV Escenario con extremas limitaciones para el & $>$ Participación funcional \\
empoderamiento & $>$ Participación consultiva \\
\hline Participación por incentivos y pasiva & $>$ Comunicación impersonal - basada en hechos \\
$>\quad$ Participación nominal & $>$ Red de organizaron informal \\
$>\quad$ Comunicación impersonal - personal & Nivel de capacitación básico & \\
$>\quad$ Inexistencia de redes de organización &
\end{tabular}

La información de los escenarios ubica a cada nivel en función del plano cartesiano. Elaboración propia. 


\section{Fase III. Elaboración de Informe}

Paso 1. Elaboración del primer borrador. En este primer momento se obtuvieron los primeros resultados de la investigación, dando así la pauta para el análisis de los primeros datos colectados en campos mediante la aplicación de los instrumentos utilizados. Esto con el fin de conocer una primera aproximación de los resultados esperados.

Paso 2. Retroalimentación. Para validar la informacion obtenida en el primer borrador del documento, se realizó retroalinetación con los actores locales de San José de Cusmapa (mujeres facilitadoras, mujeres comunitarias, tecnicos de las instituciones, responsables de programas y proyectos $\mathrm{y}$, lideres comunitarios y alcadesa municipal) en presencia de docentes de la UNA, Tesistas y responsables de la UPM, lo que permitió la continuidad del trabajo de investigación. Los actores locales involucradas en el proceso de investigacion manifestaron sus puntos de vistas acerca de la secuencia que llevaba la investigación, de acuerdo a los objetivos propuestos y los cambios que ellos hicieran permitió la continuidad del trabajo de investigacion resultando así un segundo borrador.

Paso 3: Elaboración del segundo borrador. Los datos analizados en el proceso de retroalimentacón permitio la elaboracion del documento con una mejor estructura para la presentacion del informe final.

Paso 4: Elaboración del informe final. En esta etapa se presentaron los resultados finales de los datos procesados y analizados respectivamente, de la presente investigación. Culminando de esta manera el presente trabajo de Tesis.

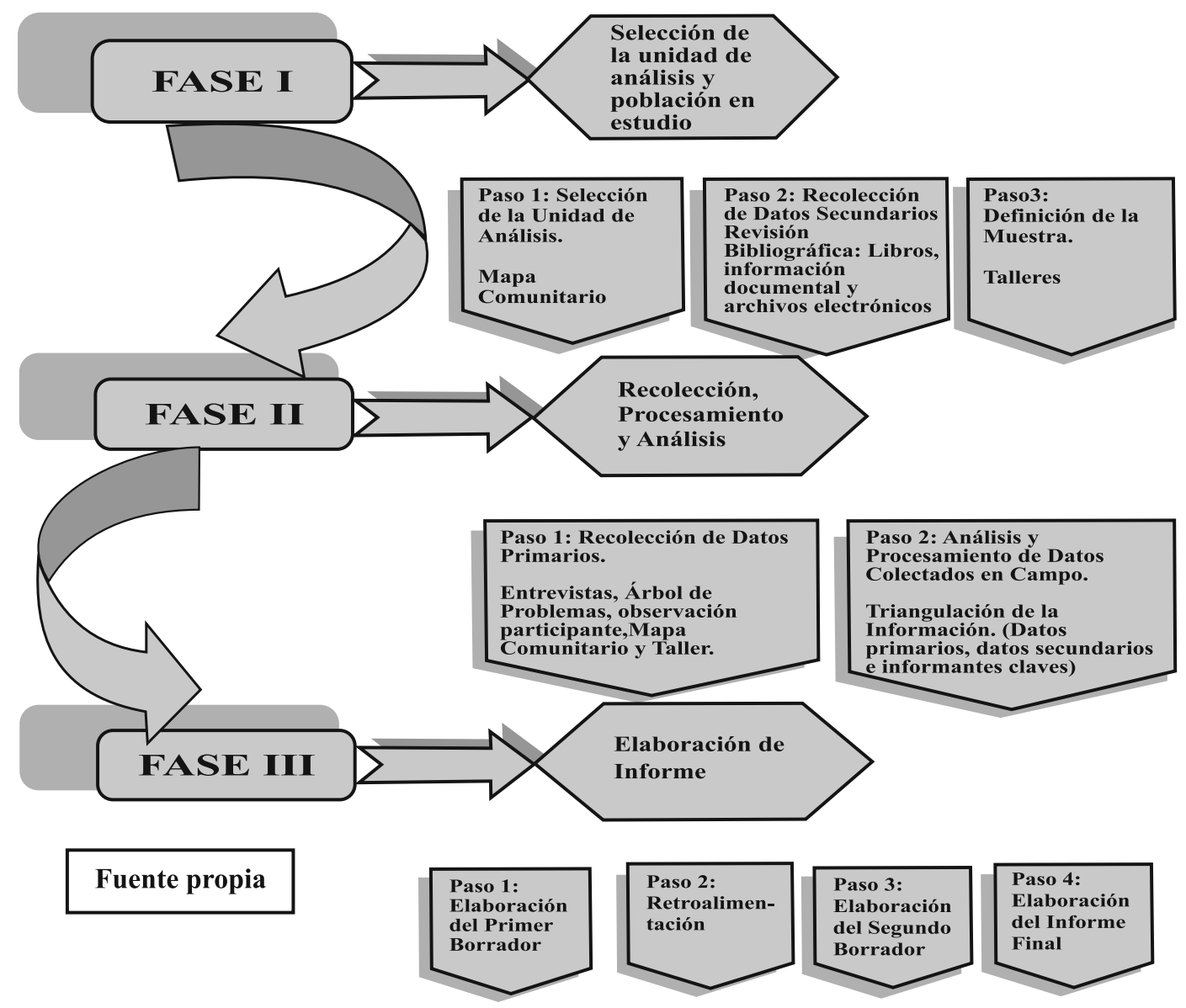

Figura 3. Esquematización del diseño metodológico 


\section{RESULTADOS Y DISCUSIÓN}

Empoderamiento de las mujeres en programas y proyectos SAN comunidad San Francisco de Imire/ proyectoacción contra el hambre -PRESANCA y comunidad Ángel 2/proyecto-movimiento comunal nicaragüense. La organización de las mujeres en las comunidades surge a partir de los criterios establecidos por los proyectos, una vez que estos se retiran, la organización comunal deja de existir.

La participación de las mujeres en las comunidades se ve afectada por falta de conciencia de los hombres al no dejarlas involucrarse en los procesos de desarrollo.

De acuerdo al análisis de los factores sociales, las comunidades se encuentran en el IV escenario, lo que indica que presentan extremas limitaciones para el empoderamiento.

Comunidad Los Llanitos/ programa-MINSA. El programa estableció como criterio principal la eficiente comunicación de las mujeres por lo que solicitaba la elaboración y difusión de los materiales de comunicación por parte de ellas y por este cumplimiento la comunidad fue elegida por el programa. De acuerdo al análisis de los factores sociales, la comunidad se encuentra en el I escenario, lo que indica que se encuentra en óptimo desarrollo de empoderamiento.

Comunidad Ángel 1/ Programa Hambre cero MAGFOR, Comunidad El Mojón/ Proyecto - INSFOP - UNICAM y Comunidad EI Mamey/ Programa - Fundación Padre Fabretto. Las mujeres de esta comunidad están en proceso de organización debido a las continuas capacitaciones que han tenido en diversos proyectos de desarrollo, por ende las decisiones deben de ser en consenso para decidir como agentes comunitarios.

La comunidad cuenta con muchas mujeres líderes donde el trabajo los realizan de manera participativa, la mayoría del tiempo lo hacen en reuniones, charlas y capacitaciones, donde interactúan y asumen control acerca de las decisiones locales que les competen. Los programas y proyectos toman en cuenta este factor ya que involucran más a mujeres que varones por su grado de responsabilidad.

De acuerdo al análisis de los factores sociales, la comunidad se encuentra en el II escenario, lo que indica que presenta oportunidades para el empoderamiento.

Comunidad El Apante/ Proyecto - PROSUD. Las mujeres que participaron en el proyecto de esta comunidad lo hicieron de manera formal, puntual, activa y responsable, adquiriendo nuevos conocimientos, que serán duplicados a otras mujeres para que se involucren en otros proyectos en ejecución.

Las mujeres de esta comunidad no mostraron interés en la conformación de la organización que el proyecto les brindo realizar durante la ejecución del mismo.

De acuerdo al análisis de los factores sociales, la comunidad se encuentra en el III escenario, lo que indica que presenta limitaciones para el empoderamiento.

Niveles de empoderamiento de las mujeres en programas y proyectos SAN. El análisis de la información permitió ubicar a las comunidades en escenarios de niveles de empoderamiento, tomando como referencia los productos esperados de las variables planteadas.

Tabla 3. Ubicación de las comunidades en escenarios de empoderamiento (Elaboración propia)

II Escenario con oportunidad para el Empoderamiento.

Ángel 1

El Mojón

El Mamey

IV Escenario con extremas limitaciones para el empoderamiento.

San Francisco de Imire Ángel 2
I Escenario óptimo para Empoderamiento.

Los Llanitos

III Escenario con limitaciones para el Empoderamiento.

El Apante 
De acuerdo al análisis de los factores sociales, la comunidad Los Llanitos se encuentra en el I escenario, lo que indica que se encuentra en óptimo desarrollo de empoderamiento. Las mujeres participan tomando la iniciativa para crear sistemas, independientemente de la institución que beneficia con el proyecto en la comunidad, además ellas tienen un papel importante en la toma de decisiones sobre qué se va a hacer, cómo y quien, trabajan de manera conjunta, se encuentran organizadas y se movilizan para solucionar problemas de interés común.

De acuerdo al análisis de los factores sociales, las comunidades Ángel 1 El Mojón y El Mamey se encuentra en el II escenario, lo que indica que presentan oportunidades para el empoderamiento.Las mujeres participan en análisis conjuntos que conducen a planes de acción y a la formación de nuevos grupos locales y al fortalecimiento de los ya existentes.Se encuentran organizadas de manera nominal y están activas, es decir articuladas, en movilización y operantes.

De acuerdo al análisis de los factores sociales, la comunidad El Apante se encuentra en el III escenario, lo que indica que presenta limitaciones para el empoderamiento. Las mujeres forman grupos para coincidir objetivos predeterminados relacionados al proyecto, esto permite el desarrollo de organizaciones sociales externamente iniciadas, el proyecto busca las ideas y opiniones de las mujeres, pero mantienen el control sobre el proceso. El proyecto proporciona información, conocimientos $\mathrm{y}$ habilidades esenciales para que se apropien de el y utiliza como método eficiente los conocimientos generados por las mujeres para luego trasmitirlosa otras beneficiarias.

De acuerdo al análisis de los factores sociales, las comunidades San Francisco de Imire y Ángel 2, se encuentran en el IV escenario, lo que indica que presentan extremas limitaciones para el empoderamiento. Esto porque las mujeres participan de manera pasiva, algunas lo hacenpor incentivos lo que conlleva a que ellas no tomen sus propias decisiones en el proceso de los proyectos de desarrollo, la información que generan las beneficiarias pertenece a los profesionales externos. Existen problemas de comunicación que estan ligados a la informalidad de trasmision de los mensajes, esto se debe a la falta de organización entre las mujeres y los comunitarios.

\section{CONCLUSIONES}

La organización, capacitación, comunicación y participación en las comunidades son factores que limitan la toma de decisiones en los asuntos que ponen en riesgo la seguridad alimentaria de las mujeres y de sus familias.

La participación de las mujeres en los programas y proyectosenlas comunidades es limitada porfaltade conciencia de los hombres de no brindar oportunidades igualitarias.

La organización comunal es fundamental para la participación de las mujeres en los programas y proyectos, esto refuerza los grupos de mujeres que trabajan de manera conjunta, si no existe este componente no hay fuerza motriz para participar en proyectos de desarrollo, sin embargo la organización de las mujeres se realiza mientras se ejecutan los programas y proyectos.

Los programas y proyectos capacitan a las mujeres en temas sobre seguridad alimentaria y nutricional (huertos familiares, elaboración de alimentos a base de hojas verdes, huertos escolares, alimentación balanceada) lo que ha contribuido el acceso al conocimiento.

Las distancias de una comunidad a otra y de estas con relación a la ubicación de los programas y proyectos, así como los mecanismos de comunicación implementados (líderes comunitarios y técnicos) ha sido un factor que limita la comunicación en las comunidades, los cuales se ligan a la distorsión y sesgos de la información, haciendo de esta manera las comunidades no efectivas.

Los niveles de comunicación en las comunidades estudiadas se refieren al personal-impersonal debido a que solo existe interaccion entre las mujeres y los técnicos de los programas y proyectos de seguridad alimentaria y nutricional, y no con los ejecutores, por lo que, los problemas de comunicación persisten en todo el proceso de comunicación.

\section{RECOMENDACIONES}

Interacción entre instituciones para fortalecer acciones en pro del desarrollo de las comunidades trabajando la temática de desarrolloorganizativoaniveldeagenciay comunidad,creando redes que le permita a los comunitarios ser autogestionarios.

Realizar diagnósticos a nivel comunitario para obtener información básica que sirva de referencia en futuros planes de acción de desarrollo, en función de garantizar la seguridad alimentaria.

La capacitación debe realizarse en función de la efectiva participación de los comunitarios de manera que permita en mediano y largo plazo la implementación de técnicas en seguridad alimentaria.

Dirigir capacitaciones con enfoque de género, que permita disminuir el impacto negativo de la efectiva participación de las mujeres en los programas y proyectos de desarrollo que se ejecutan en las comunidades de San José de Cusmapa.

Los proyectos y programas de desarrollo en las comunidades deben permitir el involucramiento de las beneficiarias en los mecanismos de trabajo y los medios de comunicación, validándolos de manera conjunta (técnicosmujeres), esto contribuirá al empoderamiento de las mujeres en todo el proceso.

La metodologia utilizada permitirá el acceso y uso a nuevosinvestigadoresmotivadoseneltemadeempoderamiento, por tanto se sugiere uilizar los materiales y metodos usados en el presente estudio. 


\section{REFERENCIAS BIBLIOGRÁFICAS}

Aguirre, B. 1995. Etnografía. Metodología cualitativa en la investigación sociocultural. ed. Boixareu universitaria. Barcelona, 1995. P. 73.

AMMA. 2006 . diagnostico departamental. (Asociación de municipios Madriz-Madrid). P. 5.

ASOCAM. 2007. Empoderamiento, conceptos y orientaciones, Guía para desarrollar estrategias y acciones en procesos orientados al empoderamiento. Publica en Quito, Octubre 2007. P. 3 y 7.

Lomonosov, BF. 2007. El problema de la comunicación en psicología, P. 89

Dabas, E. 1998. Las redes de comunicación sociales, segunda edición, Guatemala, pág. 107.

PASOLAC, UNICAM. 2001. Evaluación participativa por productores, una guía metodológica para la evaluación del efecto e impacto de desarrollo. P 9-50.

Piura, LJ. 1994. Introducción a la metodología de la investigación científica; Cuarta Edición, Publicada de la Escuela de Salud Pública de Nicaragua; P. 17-58; 121-133.

PRESANCA. 2007. Caracterización actual del municipio Cusmapa. Publicada en San José de Cusmapa, Julio 2008. P. 81. 